\title{
A CLASSICAL INSTANTON ON A FOUR-DIMENSIONAL PERIODIC LATTICE
}

\author{
I.A. FOX, M.L. LAURSEN, G. SCHIERHOLZ \\ Deutsches Elektronen-Synchrotron DESY, Hamburg, West Germany \\ J.P. GILCHRIST \\ Physics Department, University of Edinburgh, Edinburgh, Scotland, UK
}

and

M. GÖCKELER

Institut für Theoretische Physik der Universität Heidelberg, Heidelberg. West Germany

Received 11 April 1985

On a four-dimensional periodic lattice, we construct an SU(2) gauge field configuration which is analogous to the classical instanton. This is shown to have topological charge equal to one. We calculate the eigenvalue spectrum of the fermion matrix and demonstrate the existence of an approximate zero mode.

In a previous paper [1], some of us carried out a Monte Carlo study of the topological charge $Q$ in SU(2) lattice gauge theory using the construction of $Q$ given by Lüscher [2]. It was found that, on a periodic lattice of volume $V$, the topological susceptibility $\chi_{t}:=\left\langle Q^{2}\right\rangle / V$ followed asymptotic scaling, from which we concluded that our present values of $\beta$ (and lattice sizes) are relevant for continuum physics.

In order to understand the structure of the QCD vacuum, it is important to find an interpretation of topology and, in particular, to see if it can be attributed to the presence of instantons.

Moreover, it is well known that in the continuum theory with fermions, to each instanton, there corresponds one fermion zero mode [3]. On a finite lattice, with staggered fermions, these zero modes translate into small eigenvalues of the fermion matrix. (These go to zero as $V \rightarrow \infty$.) Since small eigenvalues are known to be related to chiral symmetry breaking on the lattice [4], it is not inconceivable that instantons may play a role in this phenomenon [5].

In order to study such connections, one needs a meaningful definition of a classical instanton on a pe- riodic lattice (four-torus). At first sight this seems difficult. Indeed, if one simply takes the naive discretized version of the instanton and, using some damping function (which is one in the interior of the lattice), make the gauge potential vanish at the boundary in accordance with periodicity, one obtains $Q=0$ (irrespective of whether the regular or singular gauge is used).

An analytic expression which is periodic in the time direction only has been given by Gürsey and Tze [6]. It is even possible to make the solution periodic in all four directions but, since no closed form exists, it is impractical from a numerical point of view.

In this paper, we propose a lattice definition of the instanton solution which we show leads to non-trivial topology $(Q=1)$. The essential points of the construction are as follows.

We start from the continuum instanton potential which in the regular and singular gauges may be written respectively as

$A_{\mu}(x)=\left[\mathrm{i} /\left(x^{2}+R^{2}\right)\right] \frac{1}{2}\left(s_{\mu} \bar{s}_{\nu}-s_{\nu} \bar{s}_{\mu}\right) x_{\nu}$, and 
$\tilde{A}_{\mu}(x)=\left[\mathrm{i} R^{2} /\left(x^{2}+R^{2}\right)\right] \frac{1}{2}\left(\bar{s}_{\mu} s_{\nu}-\bar{s}_{\nu} s_{\mu}\right) x_{\nu} / x^{2}$,

where $s_{4}=\bar{s}_{4}=1, s_{j}=-\bar{s}_{j}=\mathrm{i} \sigma_{j}(j=1,2,3)$ and $R$ is the "size" of the instanton. Except at the origin $x=0$, the two gauge potentials are related by a singular gauge transformation $\tilde{g}(x)=x_{\mu} s_{\mu} /|x|$.

We then map the real line into the interval $(0, L)$ by means of the coordinate transformation $x \rightarrow y$ where

$x_{\mu}=L^{3}\left[\left(L-y_{\mu}\right)^{-2}-y_{\mu}^{-2}\right]$.

We identify $L$ with the length of a four-dimensional, hypercubic, periodic lattice $T$. ( $L$ is taken to be an even multiple of the lattice spacing). The points $x_{\mu} \rightarrow$ $\pm \infty$ where the behaviour of the potential (1a) leads to a non-vanishing topological charge, correspond to $y_{\mu}$ $=0$ and $y_{\mu}=L$, i.e. they are mapped onto the boundary of the lattice.

Under the transformation (2), $A_{\mu}(x)$ and $\tilde{A}_{\mu}(x)$ are mapped to $B_{\mu}(y)$ and $\widetilde{B}_{\mu}(y)$ respectively. Except at $y_{\lambda}=L / 2(\lambda=1,2,3,4)$, the transformed gauge potentials are also related by a singular gauge transformation $g(y)$. Note that while $\widetilde{B}_{\mu}(y) \rightarrow 0$ for $y \rightarrow 0$ or $L$ (for any $\lambda \in\{1,2,3,4\}$ ), $B_{\mu}(y)$ is singular on the lattice boundary.

Although $\widetilde{B}_{\mu}(y)$ does have the correct behaviour at the boundary, if we simply considered it on the whole lattice, this of course would still lead to $Q=0$. However, if we interpolate between the two solutions, $B_{\mu}(y)$ given on some interior domain $T_{\mathrm{i}}$ of the lattice and $\widetilde{B}_{\mu}(y)$ on an outer domain $T_{\mathrm{o}}$, then it is indeed possible to get out of the $Q=0$ sector.

Explicitly, setting the lattice spacing to one,

$T_{\mathrm{i}}=\left\{y \in T \mid L / 2-M \leqslant y_{\lambda} \leqslant L / 2+M, \lambda=1,2,3,4\right\}$,

$T_{\mathrm{o}}=\left\{y \in T \mid 0 \leqslant y_{\lambda} \leqslant L / 2-M\right.$ or $L / 2+M \leqslant y_{\lambda} \leqslant L$,

$$
\text { for any } \lambda \in\{1,2,3,4\}\} \text {, }
$$

where $M \in\{1,2, \ldots, L / 2-1\}$.

If the link $(y, y+\hat{\mu})$ lies completely within $T_{\mathrm{i}}$, we set

$U_{\mu}(y)=P \exp \left(\mathrm{i} \int_{0}^{1} \mathrm{~d} t B_{\mu}(y+(1-t) \hat{\mu})\right)$,

while if it lies completely within $T_{0}$, we set

$\widetilde{U}_{\mu}(y)=\mathrm{P} \exp \left(\mathrm{i} \int_{0}^{1} \mathrm{~d} t \widetilde{B}_{\mu}(y+(1-t) \hat{\mu})\right)$, where $\mathbf{P}$ denotes path ordering. For links $(y, y+\hat{\mu})$ contained in the intersection $T_{\mathrm{i}} \cap T_{\mathrm{o}}$, we have $U_{\mu}(y)$ as well as $\widetilde{U}_{\mu}(y)$ and they are related by the gauge transformation $g(y)$. Therefore, we replace $U_{\mu}(y)$ on links completely within $T_{\mathrm{i}}$ by

$\hat{U}_{\mu}(y)=\hat{g}^{-1}(y) U_{\mu}(y) \hat{g}(y+\hat{\mu})$,

where

$$
\begin{aligned}
\hat{g}(y) & =g(y) & & \text { for } y \in T_{\mathrm{i}} \cap T_{\mathrm{o}}, \\
& =1 & & \text { for } y \in T_{\mathrm{i}} \backslash\left(T_{\mathrm{i}} \cap T_{\mathrm{o}}\right) .
\end{aligned}
$$

In this way, we achieve that $\hat{U}_{\mu}(y)=\widetilde{U}_{\mu}(y)$ on links contained in $T_{\mathrm{i}} \cap T_{\mathrm{o}}$ and get a well-defined gauge field configuration on the whole lattice. Note that $U_{\mu}(y)$ is the unit matrix if $(y, y+\hat{\mu})$ lies on the boundary. For the actual calculation the path ordered exponentials in (4a), (4b) are approximated by $\exp \left[\mathrm{i} B_{\mu}\left(y+\frac{1}{2} \hat{\mu}\right)\right]$ and $\exp \left[\mathrm{i} \widetilde{B}_{\mu}\left(y+\frac{1}{2} \hat{\mu}\right)\right]$.

We then checked numerically that this configuration does indeed have $Q=1$. All measurements were carried out for $L=6$. Using our program for measuring the topological charge [1], we calculated $Q$ for the above configuration for various values of the instanton size parameter $R(=0.5,1.0,1.5,2.0)$. In all cases we found $Q=1$.

In addition, we calculated the eigenvalues $\lambda_{i}(i=1$, $\ldots, 2 L^{4}$ ) of the fermion matrix i $M$ using staggered fermions. These eigenvalues were calculated using the Lanczos algorithm [4]. In the continuum, i $M$ corresponds to the inverse propagator at zero quark mass.

In fig. 1 we plot the density of eigenvalues $\rho(\lambda)$ for the above instanton solution. We clearly observe a small, isolated eigenvalue which we can then associate

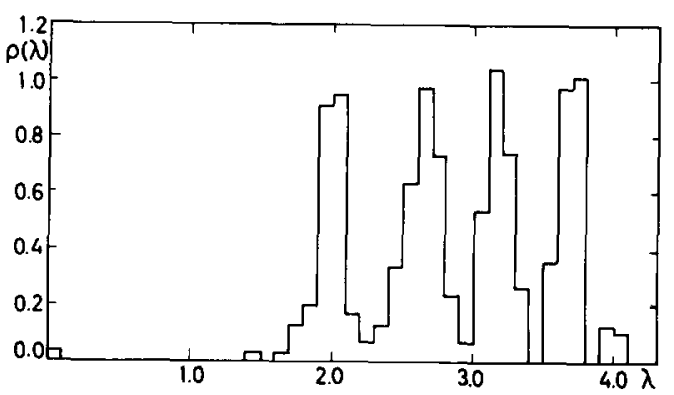

Fig. 1. The density of eigenvalues $\rho(\lambda)$ of the fermion matrix for a classical instanton solution. 
with a zero mode, as expected from the index theorem [7].

The form of the distribution, which consists of five peaks, reflects the fact that the instanton introduces a small perturbation in to the vacuum. For a gauge field configuration in equilibrium, the distribution looks completely different, namely it extends smoothly to $\lambda=0$.

Having constructed a classical instanton on the four-torus, we are now in a position to investigate the topological properties of the vacuum in detail and confront these with the usual instanton interpretation. This question will be addressed in a forthcoming paper [8].

We would like to thank $M$. Lüscher and $M$. Teper fo helpful discussions. I.A.F. would like to thank the
Royal Society and J.P.G., the SERC, for fellowships. We are grateful to the University of Hamburg for the use of their computer facilities.

\section{References}

[1] I.A. Fox, J.P. Gilchrist, M.L. Laursen and G. Schierholz, Phys. Rev. Lett. 54 (1985) 749.

[2] M. Lüscher, Commun. Math. Phys. 85 (1982) 29.

[3] S. Coleman, The uses of instantons, in: The whys of subnuclear physics (Erice, 1977), ed. A. Zichichi (Plenum, New York, 1979) p. 805.

[4] I.M. Barbour, P. Gibbs, J.P. Gilchrist, M. Schneider G. Schierholz and M. Teper, Phys. Lett. 136B (1984) 80.

[5] G. Veneziano, Phys. Lett. 95B (1980) 90.

[6] F. Gürsey and H.C. Tze, Ann. Phys. 128 (1980) 29.

[7] M. Atiyah and I. Singer, Ann. Math. 87 (1968) 484.

[8] I.A. Fox, J.P. Gilchrist, M.L. Laursen, G. Schierholz and M. Teper, in preparation. 